Témoigner Témoigner. Entre histoire et mémoire

Getuigen Revue pluridisciplinaire de la Fondation Auschwitz

Violences radicales en scène

\title{
Himmelweg de Juan Mayorga et les témoins de Claude Lanzmann
}

Daniel Weyssow

\section{(2) OpenEdition}

1 Journals

Édition électronique

URL : https://journals.openedition.org/temoigner/3403

DOI : 10.4000/temoigner.3403

ISSN : 2506-6390

Éditeur :

Éditions du Centre d'études et de documentation Mémoire d'Auschwitz, Éditions Kimé

Édition imprimée

Date de publication : 1 octobre 2015

Pagination : 135-140

ISSN : 2031-4183

Référence électronique

Daniel Weyssow, «Himmelweg de Juan Mayorga et les témoins de Claude Lanzmann », Témoigner.

Entre histoire et mémoire [En ligne], 121 | 2015, mis en ligne le 01 octobre 2016, consulté le 04 février 2022. URL : http://journals.openedition.org/temoigner/3403; DOI : https://doi.org/10.4000/temoigner. 3403

Tous droits réservés 


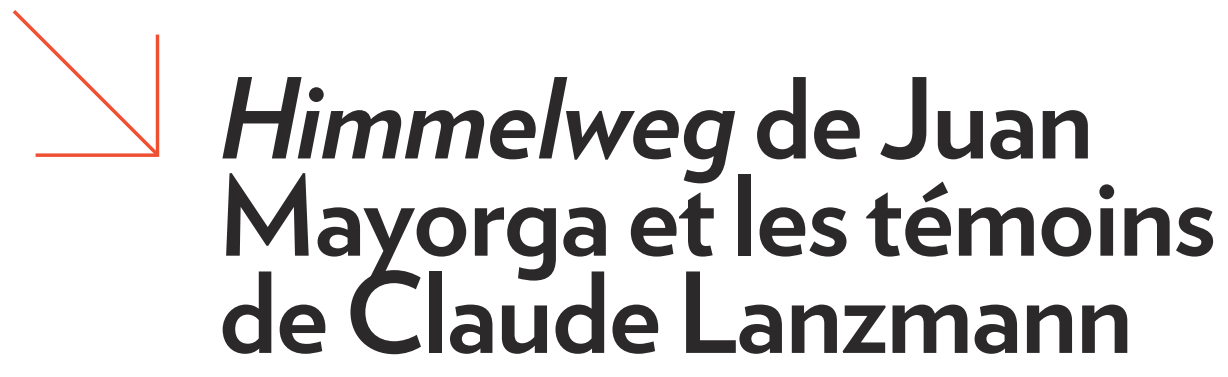

$\rightarrow$ Par Daniel Weyssow,

Fondation Auschwitz 
la Croix-Rouge). Contre toute attente, la police d'Himmler l'a laissé avancer sur les routes, fanion au vent, jusqu'au camp, où il a pu rencontrer le commandant. Il souhaitait obtenir des « renseignements » concernant les conditions de vie des prisonniers. En réponse, le commandant, qui s'est présenté à lui en philosophe humaniste et cultivé, l’a invité à visiter les lieux, en fait un ghetto.

Accompagné du commandant et du Judenälteste qui lui est présenté comme le maire de la ville, ils parcourent des rues asphaltées et fleuries, écoutent un orchestre jouer sur la place principale. Les photos sont permises. L’ambiance générale semble bonne, mais le délégué ignore qu'il s'agit d'une mise en scène organisée à son intention. Tout a été pensé et arrangé pour que ce jour de visite apaise ses inquiétudes. Il quitte le ghetto soulagé et rédige un rapport indiquant que les Juifs sont bien traités. Des années plus tard, il revient sur la mystification dont il fut l'objet. S’il est clair qu'il n'a pas tout vu, il ne se reproche rien. Il ne pouvait tout simplement pas, estime-t-il, inventer ce qu'on ne lui a pas montré. Et puis, il a trouvé les Juifs hautains et distants. Pourquoi ne lui ont-ils rien dit? «Toutes les nuits, ma mémoire écrit et réécrit : "Les conditions d'hygiène sont satisfaisantes. Les personnes sont correctement vêtues, avec des différences logiques dues aux classes sociales et aux zones de provenance. Les conditions d'hébergement sont modestes mais dignes. La nourriture parait suffisante.” » (Mayorga, 23)

La pièce s'anime, le ghetto apparaît. Des projections montrent des enfants qui jouent à la toupie. On ne sait jamais vraiment où le mouvement giratoire risque de l'emporter, mais à force de répétitions, l'objet semble sous contrôle. D'autres enfants se promènent ou se baignent dans la rivière. Assis sur un banc, un jeune homme offre un cadeau à son amie. De loin, leur attitude est conforme à ce que le commandant attend d'eux, mais à l'abri de toute écoute, ils s'entretiennent de leur désarroi et du sort qui certainement les attend.

Rideau. Second acte. Cette fois, c'est le commandant qui se remémore la présence de l'émissaire de la Croix-Rouge. Il récapitule la prestation qu'il lui a servie, à coup de références philosophiques, à son arrivée. « Je sais, vous n'êtes pas venu voir des livres, vous êtes impatient de commencer la visite. Je sais ce qui vous a poussé à faire le voyage. Inutile de me le dire : la rumeur est venue jusqu’à moi. On colporte des monstruosités. » (Mayorga, 40) Il détaille ensuite le travail accompli avec le Judenälteste pour mettre au point la mise en scène destinée à le tromper.

Par l'effet d'un nouveau tourbillon d'images projetées, de voix et de chants, nous reprenons la visite du ghetto aux côtés du délégué, du commandant, du Judenälteste et des déportés-acteurs. Chacun reste dans son rôle. Aucune improvisation n'est envisageable. Le trompe-l'œil imaginé couvre d'un voile cynique et sournois la réalité du camp. Le délégué ne pose pas de questions. Ces alertes de fin du monde ne sont plus pour lui que de lointains feux-follets d'opérette, c'est peu dire. Il ne sait pas que sept mille cinq cents personnes viennent d'être déportées vers Auschwitz et Treblinka pour qu'il ne s'aperçoive pas de la surpopulation qui régnait alors. $\mathrm{Ne}$ percevant rien du subterfuge qu'il est seul à ignorer, il ne conçoit, semble-t-il, absolument rien du piège mortel dans lequel baignent les déportés du ghetto. En vérité, 


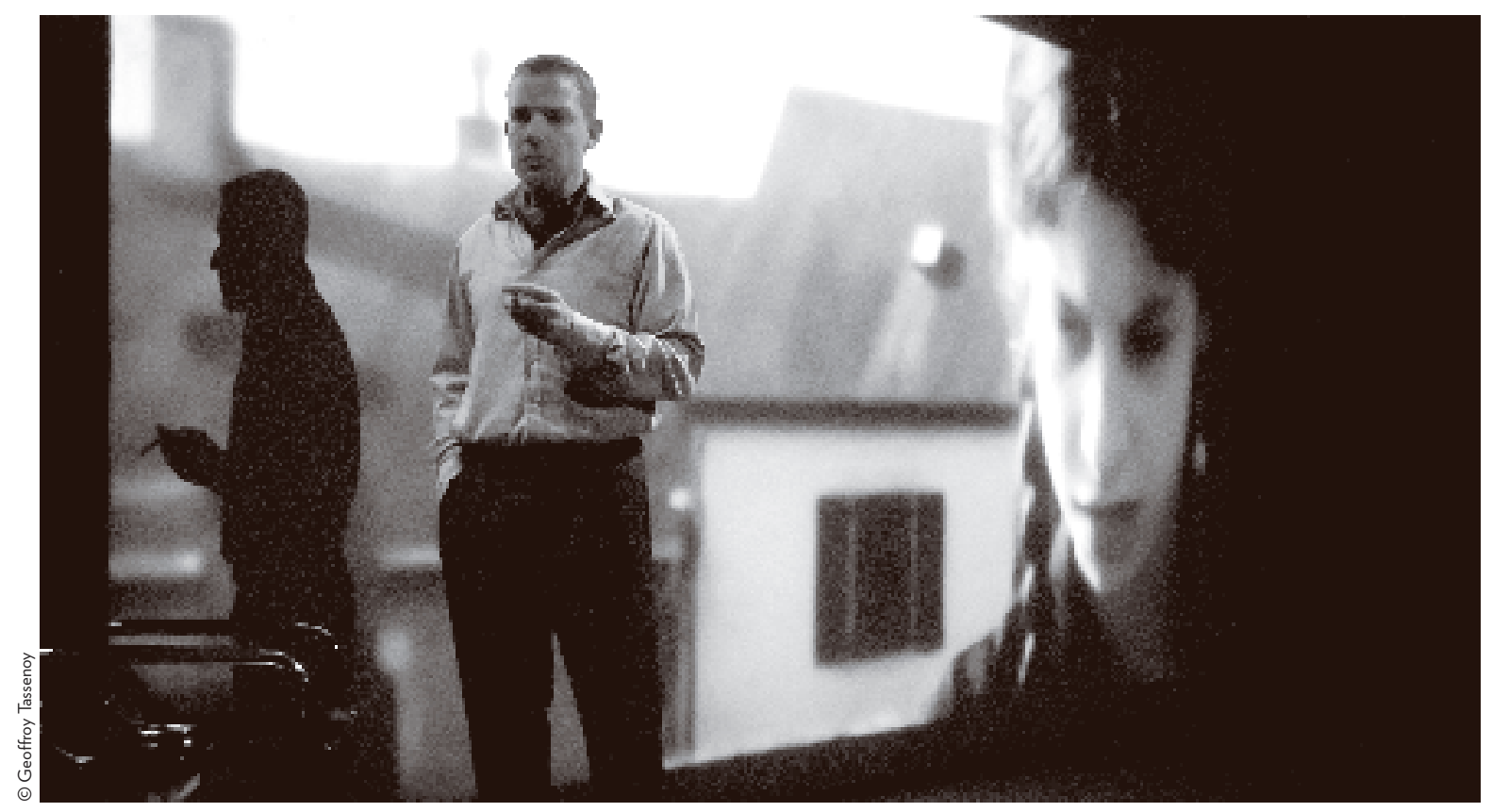

_Himmelweg.

la faim et le désespoir sont omniprésents, la moindre broutille mène à la potence, et les déportations se poursuivent en cadence.

Des images fortes rythment la pièce. À l'exemple de l'horloge à balancier de la gare du camp dont les rouages sont bloqués. On peut imaginer qu’à l'ouverture des portes du train, le pied à peine posé sur la rampe, les déportés subissent de plein fouet la désolation d'un temps mis à l'arrêt, synonyme d'éternité et de mort.

Avant de nous juger rappelez-vous que nous sommes en train de résoudre un problème qui a tourmentél'Europe pendant des siècles. Nous avons été les premiers à nous rendre compte qu'il s'agissait d'un problème de transports. Notre plus grand mérite est d'avoir résolu ce problème technique. D’autres l'avaient rêvé, nous l'avons fait. Vous les entendez ? Vous entendez les trains? Tous les trains d'Europe ont cette gare pour terminus. Si vous voulez les voir, il faudra passer la nuit; tous les trains arrivent au petit matin à six heures. (Mayorga, 43-44)

Le Judenälteste espère que le délégué aura la sagesse ou l'intelligence d'attendre l'arrivée du prochain transport. Il verrait alors que le chemin qu'empruntent à leur arrivée les déportés mène à l'infirmerie. L'Himmelweg du commandant aboutit en réalité aux crématoires. Mais au fond, aucun des protagonistes ne semble vraiment vouloir s'abstraire des leurres et mensonges qu'ils instaurent ou subissent.

Mayorga arrime le spectateur au contexte. Au paroxysme de l'enfermement et du non-sens, l'horreur, spectrale dans son apparent effacement, glace les sangs. L'auteur se proclame conteur et non historien. Il lui importe que le spectateur, lors 
de la représentation de la pièce, soit pris à partie, qu'il vive une expérience inédite. L'expression de la duperie est sensible et subtile à chaque instant de la pièce. Mais tout de même, on ne peut s'empêcher de se demander comment les déportés ont pu se prêter à un tel exercice de simulation-dissimulation, à une telle mise en scène. Comme au théâtre, cela suppose un scénario, des dialogues, des répliques, des comportements, des vêtements, un décor, des répétitions... Tout cela a pourtant bien eu lieu. Le récit laisse le spectateur songeur en ne répondant pas à ces questions. S’adressant au Judenälteste : «À partir de demain, mes hommes se promèneront en civil dans le camp. Ils se comporteront comme s’ils étaient en visite. Les tiens doivent s'habituer à ce genre de visiteurs, susceptibles d'ouvrir une porte au hasard et de poser n'importe quelle question. » (Mayorga, 64)

En définitive, si l'on veut comprendre l'histoire du ghetto de Theresienstadt et ses artifices, il est nécessaire, au-delà du récit parfaitement maîtrisé de Mayorga, de s'en reporter au témoignage de Benjamin Murmelstein, depuis peu à notre disposition grâce au film de Claude Lanzmann, Le Dernier des injustes. Le témoin y décrit en détail et avec passion la création et le fonctionnement de cette cité « modèle ».

Les responsables SS, partout où ils s'installaient en vue d'organiser les déportations, mettaient en place des Conseils représentatifs de la communauté juive destinés à jouer les intermédiaires. Ces derniers devaient servir à transmettre et faire respecter les ordonnances et décisions de l'occupant. Il en allait de même à Theresienstadt où les Judenältesten, placés « entre le marteau et l'enclume », selon l'expression de Murmelstein dans le film, devaient sans cesse procéder à des arbitrages pour le moins pénibles, à l'exemple de la constitution des listes de transports.

Murmelstein fut le dernier des trois Judenältesten de Theresienstadt. Le premier ayant été Jacob Edelstein, le second Paul Eppstein. Murmelstein témoigne dans le film du fait qu'il avait eu affaire à Eichmann avant la guerre. Il l'avait aperçu, barre de métal en main, à la tête du commando qui avait détruit la grande synagogue de Vienne lors de la Nuit de Cristal en novembre 1938. Peu après, il obligea Murmelstein, Grand rabbin de Vienne, rappelons-le, à devenir son conseiller en matière migratoire. Plusieurs plans d'évacuation des Juifs avaient en effet été imaginés par Eichmann (vers Madagascar, puis la Colombie et enfin la Palestine). Aucun de ces projets n'aboutit vraiment, mais des candidatures furent ouvertes. Les Juifs qui souhaitaient émigrer (avant le début de la guerre) durent se défaire de l'ensemble de leurs biens au profit des services d'Eichmann. Il s'agissait en définitive avant tout de les dévaliser. D’après Murmelstein, il décida ensuite [dans la foulée d’Heydrich] d'investir Theresienstadt pour en faire un ghetto « modèle » avec l'aide du commandant du camp. Car permettre qu'il soit un jour visité à ce titre par la Croix-Rouge détournerait l'attention de l'extermination en cours. Mais il fallait pour cela rendre la ville et ses Juifs présentables.

Murmelstein raconte pourquoi, avec son prédécesseur Eppstein, ils s'engagèrent à réaliser le projet d'Eichmann. Ils partaient du constat que les déportés, déprimés, affaiblis, ne jouissant d'aucune liberté, menacés de pendaison pour la moindre broutille, ne pouvaient pour ces raisons entretenir le ghetto. Or s'il tombait en ruine - il y 
avait déjà des poux et l'on commençait à signaler des cas de typhus -, il paraissait certain qu'il ne serait pas maintenu. La mort serait en ce cas certaine pour tous. Murmelstein et Eppstein proposèrent dès lors aux Allemands les transformations qu'ils jugeaient indispensables pour atteindre cet objectif, et leur initiative fut acceptée. Des matériaux destinés à l' « embellissement »de la ville leur furent livrés, principalement du bois et du verre pour aménager notamment des « magasins ». Murmelstein imposa des journées de travail de 10 heures, et permit les sorties le soir pour assister aux activités culturelles développées par les déportés eux-mêmes (théâtres, films, concerts, conférences).

La pièce et le film font état du fait que le délégué de la Croix Rouge, en visite en juin 1944, prit de nombreuses photographies. Celles qui ont été retrouvées donnent une idée de ce que fut le ghetto « embelli ». Il faut donc garder à l'esprit, en les regardant, qu'elles dissimulaient parfaitement les fondements et la finalité de la déportation. Les mises en scène, les « faux

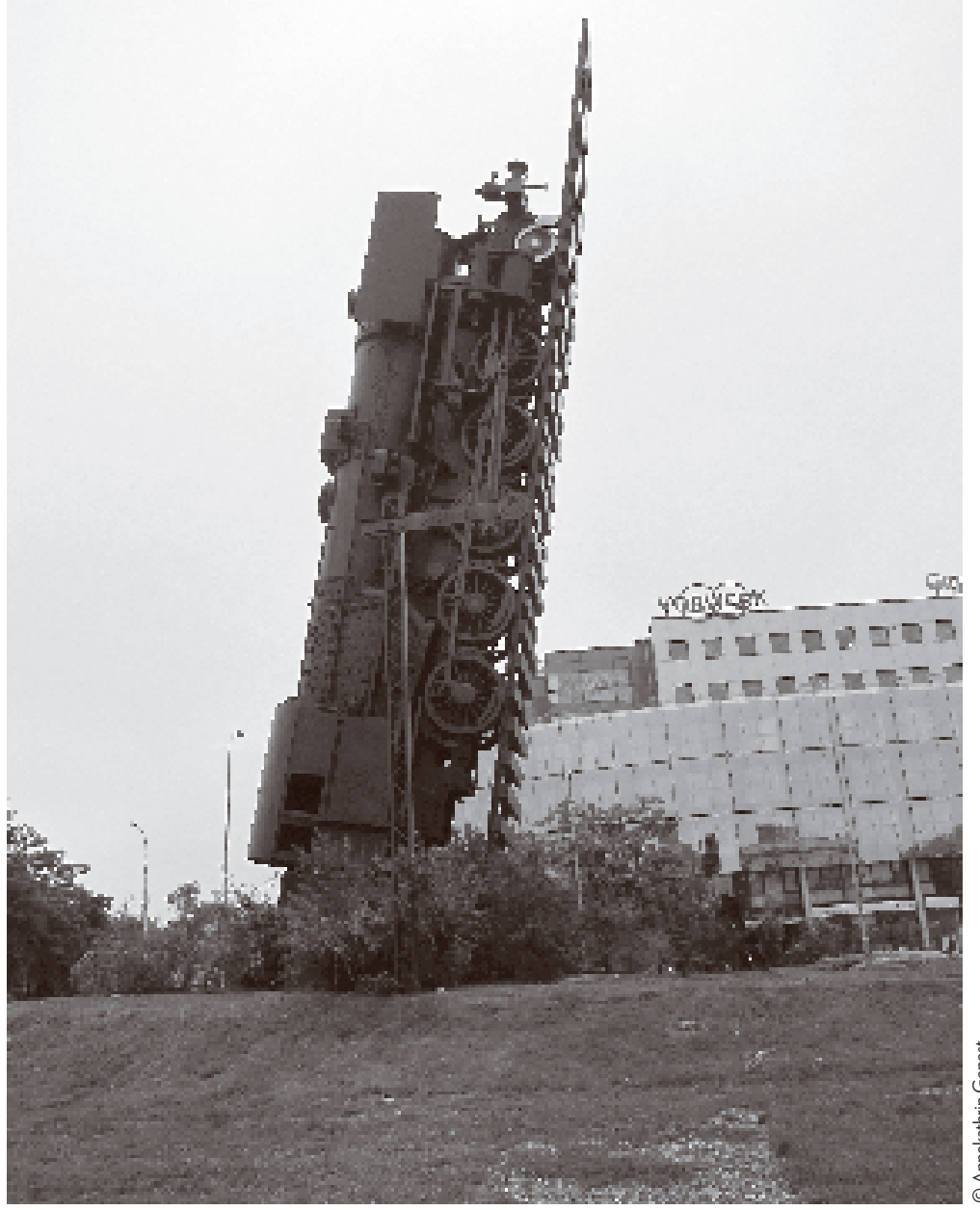

_Le Train vers le paradis /

The train to Heaven.

Monument d'Andrzej Jarodzki à Wroclaw (Pologne, 2010). Musée d’art contemporain. cateurs mentionnaient des lieux inexistants. Avec le recul, on peut affirmer que le comble de la mise en scène est atteint avec cette photographie du 23 juin 1944 où Rossel figure devant le kiosque de la place où joue, devant un public assis, un orchestre (Farré, Schubert, 2009). Outre les photographies, deux films y ont été réalisés. Il ne subsiste du premier, intitulé Theresienstadt 1942, que quelques images. Le second, filmé par Kurt Gerron (qui sera déporté un mois plus tard à Auschwitz où il sera gazé) dont il reste des fragments, a été réalisé en 1944 et terminé en 1945. Claude Lanzmann en propose des extraits, munis du son original, dans Le Dernier des injustes. Murmelstein dit avoir détesté ce film parce que les figurants y chantent, ôtant toute vraisemblance à leur comportement. 
La manipulation de l'image relève du domaine de la propagande. S’il faut à ce titre se méfier de ce qui est donné à voir, la pièce incite également à réfléchir aux raisons qui peuvent pousser la victime à devenir, en quelque sorte, complice de son bourreau. Les expériences vécues durant le III ${ }^{\mathrm{e}}$ Reich ne sont de ce point de vue certes pas uniques (pour ne citer qu'un seul autre exemple de relations captives entre otages et bourreaux, rappelons le récit de François Bizot qui, prisonnier des Khmers Rouges au Cambodge en 1971, se retrouva sous la domination directe de Douch, un des lieutenants de Pol Pot). Comment comprendre qu'il soit possible de faire le jeu de son pire ennemi jusqu'à la mort ? La réponse tient dans l'espoir de survivre, qui subsiste jusqu'au dernier souffle. Murmelstein raconte qu'il s'efforçait de se comporter à Theresienstadt comme Shéhérazade dans le conte des Mille et une nuits. Il importait de rester créatif afin de garder le bourreau en haleine en lui tricotant le décor de ses rêves.

Plus de cent quarante mille personnes furent déportées à Theresienstadt. Trentetrois mille moururent sur place et quatre-vingt-huit mille furent déportés dans les camps d'extermination. Seuls dix-neuf milles des internés de ce ghetto « modèle » survécurent à la guerre.

\section{BIBLIOGRAPHIE}

- Bizot, François (2000) : Le Portail, Paris, Éditions de la Table Ronde.

- CICR (éd.) (1990) : Documents du Comité international de la Croix-Rouge concernant le ghetto de Theresienstadt, Genève, [sans éditeur].

- Farré, Sébastien, Schubert, Yan (2009) : «L'illusion de l'objectif. Le délégué du CICR Maurice Rossel et les photographies de Theresienstadt », Le Mouvement social, 2009/2, n² 227, p. 65-83. (consultable sur internet : http://www.cairn.info/zen.php?ID_ARTICLE=LMS_227_0065).

- Mayorga, Juan (2003) : Himmelweg. Camino del cielo (publié en français sous le titre Himmelweg, traduit de l'espagnol par Yves Lebeau, Besançon, Les Solitaires Intempestifs, « Mousson d'été », 2006).

- Murmelstein, Benjamin (2013) : Terezin, il ghetto modello di Eichmann [1961], nouvelle édition avec une postface de Wolf Murmelstein (Benjamin Murmelstein, « II testimone mai sentito »), Brescia, La Scuola.

- «Rapport de Rossel suite à sa visite au K. Z. Auschwitz », 29 novembre 1944, in CICR (éd.) (2001), Chronologie de l'activité de Maurice Rossel, délégué du CICR, durant la Seconde Guerre mondiale, Genève, [sans éditeur], non paginé. Le rapport a également été publié dans la Revue d'histoire de la Shoah, n ${ }^{\circ}$ 172, mai-août 2001, p. 63-65.

\section{RÉFÉRENCES FILMOGRAPHIQUES}

- Le Dernier des injustes de Claude Lanzmann, Synecdoche, France, 2013, 218 min.

- Un Vivant qui passe de Claude Lanzmann, Les films Aleph, France, 1997, 65 min.

- Theresienstadt. Ein Dokumentarfilm aus dem jüdischen Siedlungsgebiet [Theresienstadt. Un film documentaire sur une zone de repeuplement juif], Kurt Gerron, Karel Pečený, 1945. 